\title{
Plaque rupture with severe pre-existing stenosis precipitating coronary thrombosis Characteristics of coronary atherosclerotic plaques underlying fatal occlusive thrombi
}

\author{
ERLING FALK
}

From the Institutes of Pathology, Randers Centralsygehus and Aalborg Sygehus, Denmark

SUMMARY Ruptured atheromatous plaques were identified by step-sectioning technique as responsible for $\mathbf{4 0}$ of 51 recent coronary artery thrombi and 63 larger intimal haemorrhages. The degree of pre-existing luminal narrowing at the site of rupture was decisive for whether plaque rupture caused occlusive thrombosis or just intimal haemorrhage. If the pre-existing stenosis was greater than $90 \%$ (histologically determined) then plaque rupture nearly always caused occlusive thrombosis. Clearly indicating the primary role of plaque rupture in thrombus formation were the frequent finding of plaque fragments deeply buried in the centre of the thrombus and the nature of the thrombus at the site of rupture where it consisted predominantly of platelets. Thus, a severe chronic stenosis seems to be a prerequisite for occlusive thrombus formation, but the thrombotic process itself is triggered by an acute intimal lesion.

No general agreement exists on the pathogenesis of early atherosclerotic lesions, but there seems to be little doubt that incorporation of mural thrombi ("encrustation") contributes to the progression of the atherosclerotic plaques causing luminal narrowing, ${ }^{12}$ and the final vascular occlusion is in any case always the result of a complicating thrombosis. ${ }^{3}$ The triggering mechanism responsible for a sudden thrombotic occlusion of a vessel which has been diseased for years is, however, still not well understood. Thrombus formation involves the interplay of the vascular wall with cellular and humoral factors within a flowing stream of blood. For more than 100 years three factors, usually known as the triad of Virchow, have been considered essential in thrombogenesis: abnormalities of the vessel wall, alterations of blood flow, and changes in the composition of the blood. In fatal atherosclerotic heart disease, necropsy studies have shown that coronary artery thrombosis practically never occurs unless the following are present.

(1) An acute intimal lesion underlying the thrombus. Intimal haemorrhages were once considered the primary cause of coronary thrombosis, ${ }^{45}$ but more

Accepted for publication 16 March 1983 recently the acute vascular lesions at the site of thrombosis have been described as intimal fissures, breaks, tears, rents, erosions, ulcers, and ruptures. ${ }^{6-12}$

(2) A pre-existing atherosclerotic plaque at the site of thrombosis causing severe luminal narrowing 11314 and resulting in local flow disturbances. Recent angiographic studies of patients with evolving myocardial infarction have shown that after successful intracoronary thrombolysis an underlying high grade atherosclerotic stenosis remains. ${ }^{15}$

Concerning changes in the composition of the blood, several studies have described abnormalities in platelet function, ${ }^{1617}$ coagulation factors, ${ }^{1819}$ the fibrinolytic system, and blood viscosity ${ }^{20}$ in patients with ischaemic heart disease, but these alterations may just reflect the well established fact that a thrombotic and hypercoagulable state often develops secondarily to vascular and/or myocardial lesions. ${ }^{21}$

Rupture of atherosclerotic plaques has, in addition to fatal coronary thrombosis, been held responsible for larger intimal haemorrhages, 2223 but exact knowledge of the determining factors for the ultimate outcome of a plaque rupture is lacking. Therefore, it was decided to undertake a thorough study of coronary thrombi and larger intimal haemorrhages in an 
attempt to assess the individual significance and interplay of plaque rupture and pre-existing luminal narrowing in the pathogenesis of fatal coronary thrombosis.

\section{Subjects and methods}

Forty-nine patients with clinically suspected fatal ischaemic heart disease were selected for postmortem coronary angiography at the Institute of Pathology, Randers Hospital during the period 1 February 1980 to 30 June 1981.

No appreciable coronary atherosclerosis was found in two patients, where necropsy showed a congestive cardiomyopathy in one case and a myeloproliferative disorder with acute coronary thrombosis in the other.

Coronary atherosclerosis was the underlying cause of death in the remaining 47 cases (37 men, 10 women, age range: 42 to 87 years, median age: 69 years). In three patients who died instantaneously without preceding symptoms, the necropsies disclosed a total of 10 old coronary occlusions but no recent thrombi. In 44 patients, of whom two died instantaneously while 42 had chest pain before death, the necropsies disclosed 29 old coronary occlusions and 51 recent coronary thrombi. The ages of the recent thrombi judged from the clinical symptoms and the myocardial infarcts (when present) were as follows: 26 were less than 24 hours old, 14 were 1 to 7 days old, five were 1 to 2 weeks old, and six were more than 2 weeks old.

These 47 cases of fatal atherosclerotic heart disease form the basis for this study. Eleven of them were used in a previous study, where the methods of heart examination were described in detail. ${ }^{24}$ Briefly, after postmortem coronary angiography, fixation, and decalcification, the coronary arteries were crosssectioned at $3 \mathrm{~mm}$ intervals. All segments, which angiographically or after sectioning showed occlusions, stenoses, or larger intimal haemorrhages, were processed for microscopy. All thrombosed segments were step-sectioned at $200 \mu$ intervals and the point of maximal luminal reduction resulting from old atherosclerotic plaques was identified. All larger intimal haemorrhages were also step-sectioned, though with variable intervals. Approximately 8000 sections were examined microscopically.

\section{DEFINITIONS}

Histological stenosis: the ratio between the crosssectional area of the residual lumen and the assumed original cross-sectional luminal area of the vessel (the area encircled by the internal elastic membrane).

All cross-sectional areas (A) were calculated from measurements of maximal (D) and minimal (d)



Fig. 1 Two cases illustrating plaque rupture with grossly discernible intimal haemorrhage but without significant luminal thrombosis. The vascular lumen is filled with contrast medium (Masson's trichrome stain). ( $A, B)$ No mural thrombosis is detectable by light microscopy at the site of rupture (arrow) (original magnifications $A \times 7, B \times 36) .(C, D)$ In this case, however, microscopy shows that the rupture has been sealed by a thin mural thrombus (arrow) (original magnifications $C \times 9, D \times 91$ ). 
diameters assuming an elliptical cross-section, $\mathrm{A}=1 / 4 \times \pi \times \mathrm{D} \times \mathrm{d}$ (measured by projection microscopy using a Reichert Lanometer).

Occlusive thrombus: recent thrombus obliterating at least $90 \%$ of the residual lumen.

Plaque rupture: disruption of the fibrocellular "cap" covering a pool of soft extracellular fatty material with cholesterol clefts resulting in direct contact between the flowing blood and the atheromatous "gruel".

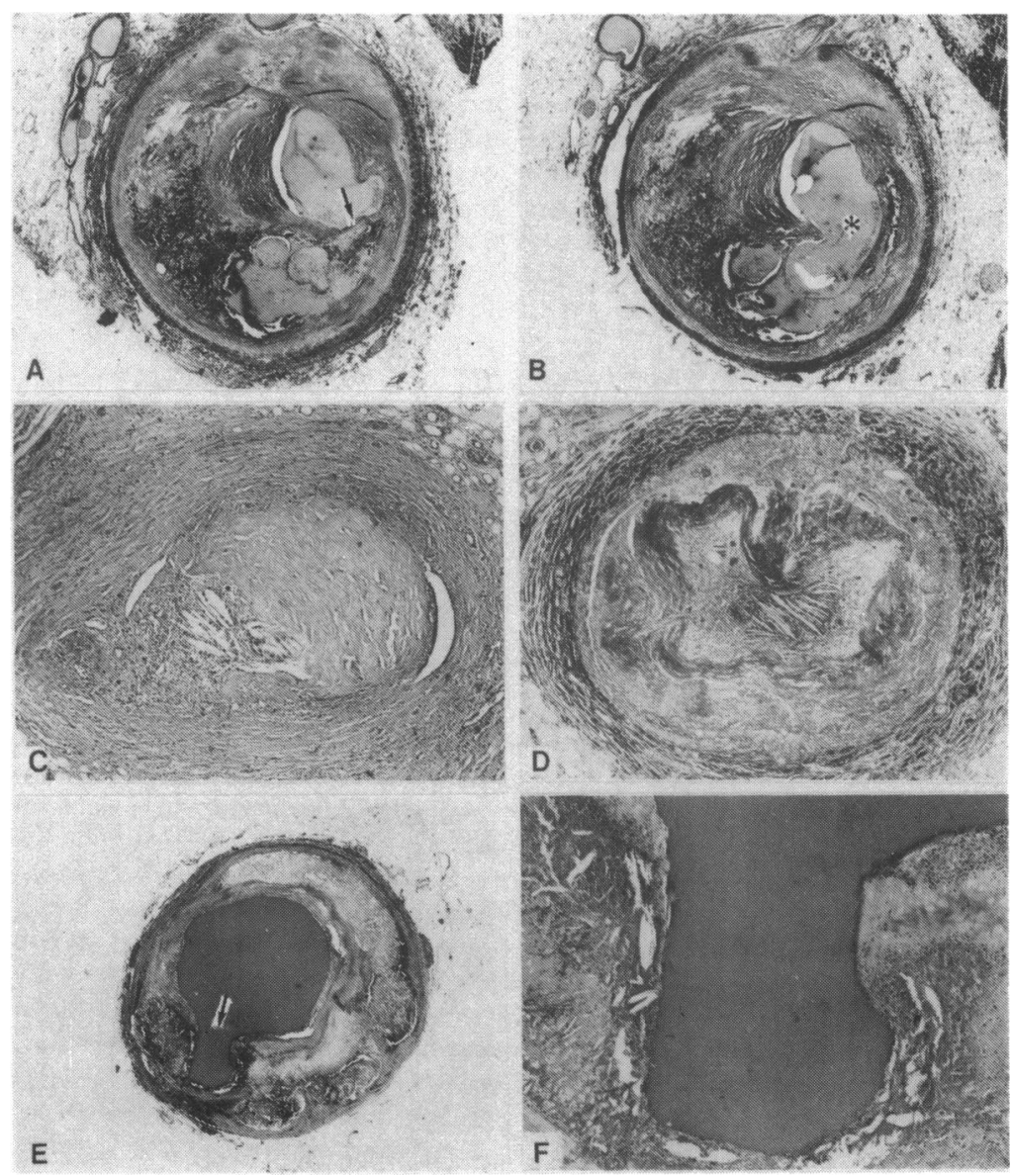

Fig. 2 Two cases illustrating plaque rupture with intimal haemorrhage and loss of some plaque material. The lost material must have been discharged into the lumen and washed away (embolised). ( $A, B) A$ fairly recent ruptured atheromatous plaque proximally in the left manginal branch. The thin fibrous cap seen in $A$ (arrow) is disrupted and partly lost in $B$ (asterisk) resulting in a wide direct communication between the flowing blood and the soft atheromatous material of the plaque. Much of the atheromatous substance has been lost (washed away) with haemorrhage into the surrounding plaque contents (original magnification $\times 15)$. (C) A small peripheral extramyocardial branch of the left marginal branch showing an older organised occlusion with cholesterol clefts, probably an organised atheroembolus (original magnification $\times 33$ ). (D) In the same patient a peripheral branch of the left anterior descending artery was occluded by fibrous tissue containing cholesterol clefts, probably an organised atheroembolus. Proximally in the artery an old plaque rupture with lost atheromatous substance was found (original magnification $\times 33) .(E, F)$ In this case microscopy of a grossly discernible intimal haemorrhage discloses a niche in the vessel wall (arrow) surnounded by intimal haemorrhage. The lost atheromatous substance must have been discharged into the lumen and washed away with the blood (embolised) (original magnification $E \times 6, F \times 33$ ). 


\section{Results}

The pathological findings are illustrated in Fig. 1 to 4 and summarised in Tables 1 to 3 .

A total of 103 plaque ruptures was identified (Table 1). Sixty three of these were associated with grossly discernible intimal haemorrhage without luminal thrombosis or with a tiny mural thrombosis sealing the rupture site which could just be seen under the microscope (Fig. 1). In several cases fatty atheromatous material had been discharged into the lumen and washed away (embolised) leaving a niche in the vessel
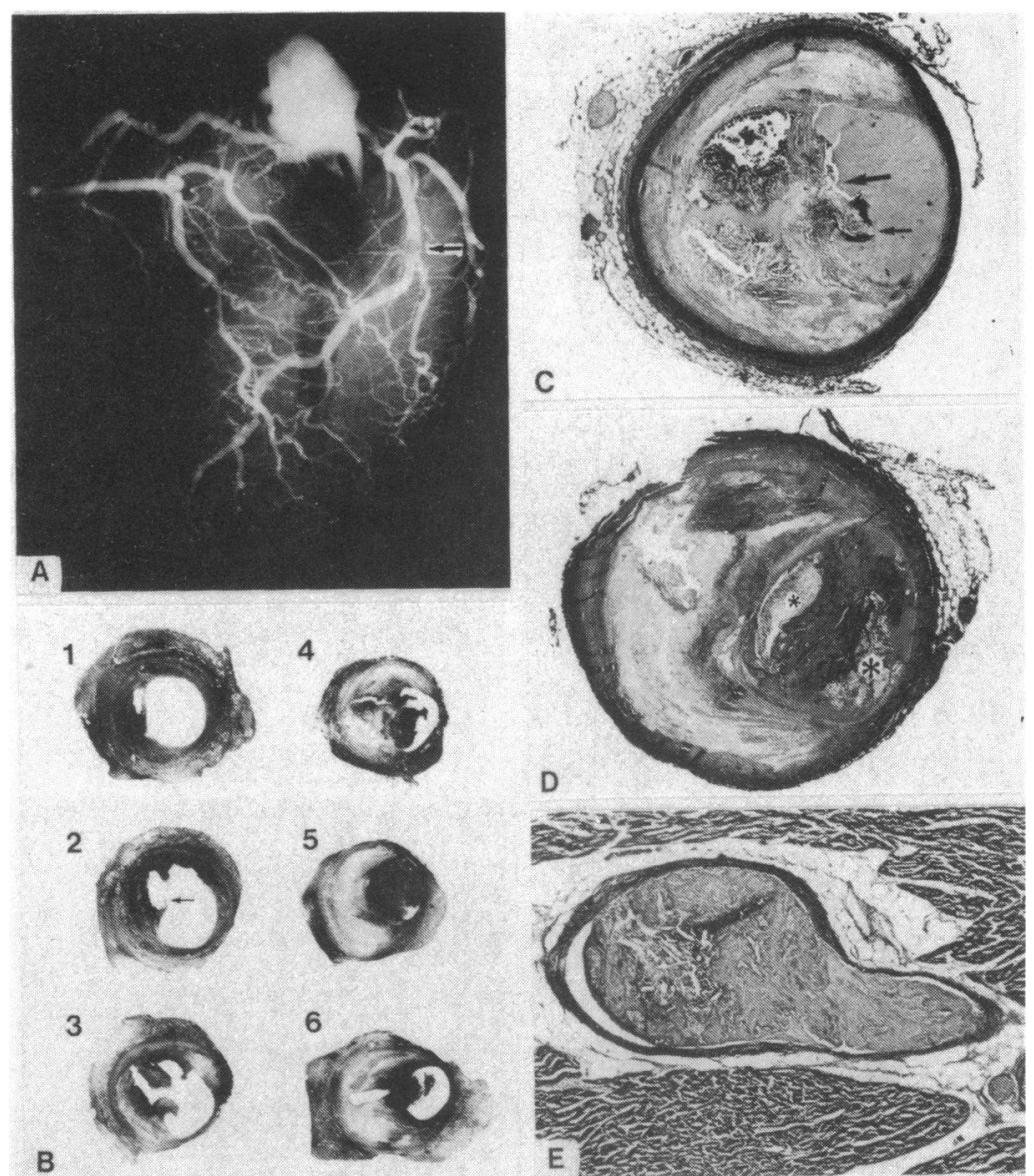

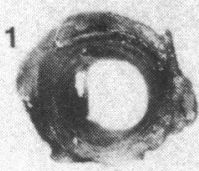

4

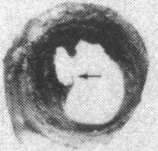

3

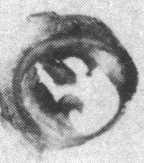

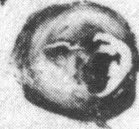

5

6
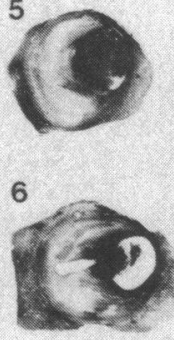

B

Fig. 3 A case clearly illustrating the primary role of plaque rupture in the pathogenesis of occlusive coronary thrombosis. (A) Postmortem angiogram showing total occlusion of the left anterior descending artery at the arrow (original magnification $\times 0 \cdot 6)$. (B) The thrombosed vascular segment is cut transversely at 2 to $3 \mathrm{~mm}$ intervals (white contrast medium in the non-thrombosed vascular lumen). Section No. 2 shows the disrupted fibrous cap (arrow), and it is seen that both a cap fragment and fatty atheromatous substance have been lost. The thrombotic process has started at the site of rupture (sections No. 2, 3, and 4) and attains its occlusive property just distal to the rupture (section No. 5). (C) Microscopy of section No. 4 shows direct communication between the vascular lumen and the atheromatous "gruel" (big arrow) and mural thrombosis at one of the free edges of the torn fibrous cap which projects into the lumen (small arrow) (original magnification $\times 13$ ). (D) Microscopy of section No. 5 shows a detached fragment of the fibrous cap (small asterisk) and atheromatous material with cholesterol clefts (big asterisk) intimately mixed with aggregated platelets in the vascular lumen (original magnification $\times 15$ ). (E) Histological section from the perfusion area of the left anterior descending artery showing an intramyocardial artery occluded by a platelet embolus containing atheromatous plaque material with cholesterol clefts from the ruptured plaque proximal in the artery (original magnification $\times 33$ ). 


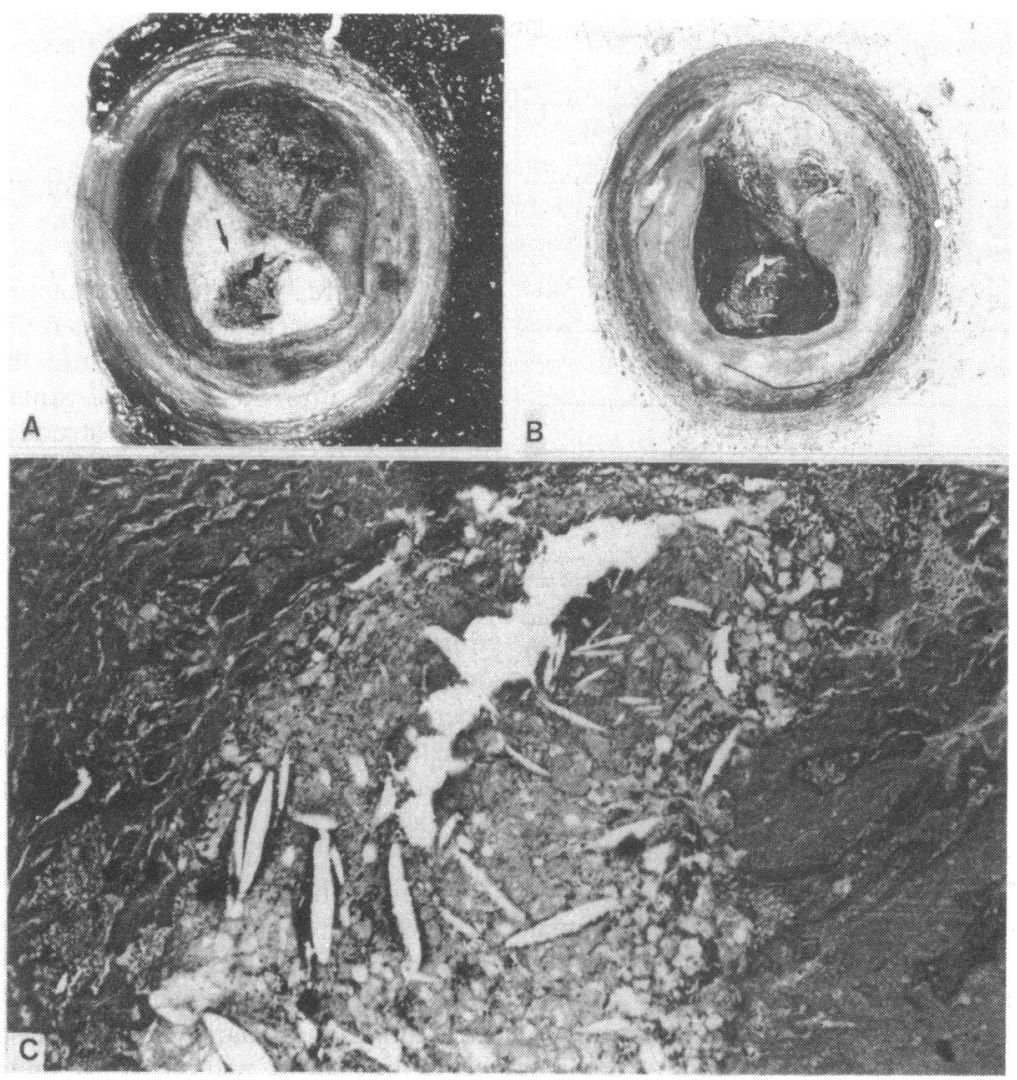

Fig. 4 Another case illustrating that plaque rupture precedes and initiates the thrombotic process in the vascular lumen. (A) Photograph of fat stained (Oil Red O) cross-sectioned coronary artery showing the lumen totally occluded by a thrombus. The fatty material (black in photograph) which is seen deeply buried in the thrombus (arrow) was first recognised grossly after application of the fat stain. $(B, C)$ Microscopy shows a plaque rupture underlying the occluding thrombosis. Fatty atheromatous plaque contents with cholesterol clefts is seen intimately mixed with the platelet thrombus (original magnifications $B \times 10, C \times 145$ ).

wall (Fig. 2). In addition to intimal haemorrhages of variable degree, there were 40 cases associated with significant luminal thrombosis (Fig. 3 and 4 ) which in all but two were occlusive (two left main stem lesions with mural thrombosis). Table 1 shows that plaque rupture was always accompanied by occlusive thrombus formation if the pre-existing stenosis was greater than $95 \%$. Rupture of plaques causing 90 to $94 \%$ stenoses was very often accompanied by occlusive thrombosis (15 of 19 cases $(79 \%)$ ), while this rarely

Table 1 Degree of pre-existing atherosclerotic stenosis at site of plaque rupture and presence or absence of complicating occlusive thrombosis

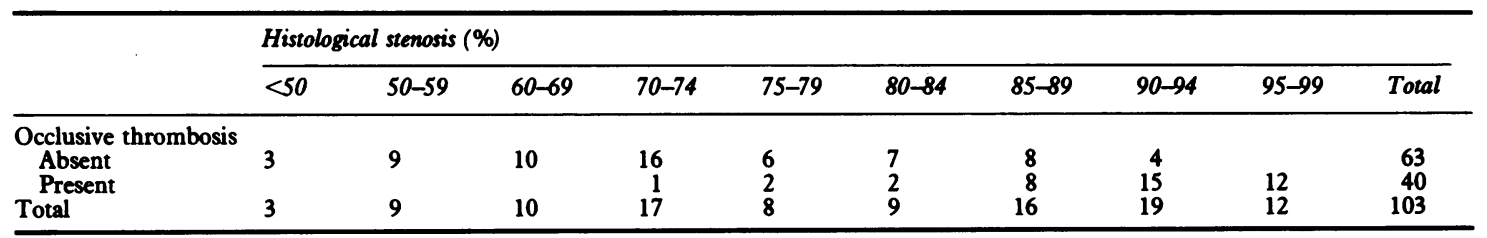

Note: Two fatal left main stem lesions ( 78 and $86 \%$ pre-existing stenosis) with mural thrombosis obstructing one-half and one-quarter, respectively, of the residual lumen are included in the group of occlusive thrombi. 
Table 2 Number and percentage of thrombi precipitated by plaque rupture correlated with age, sex, heart weight, and diabetes mellitus

\begin{tabular}{|c|c|c|c|}
\hline & & No. & $\begin{array}{l}\text { Thrombi precipitated by } \\
\text { plaque rupture }\end{array}$ \\
\hline Age (y) & $\left\{\begin{array}{l}40-49 \\
50-59 \\
60-69 \\
70-79 \\
80-89\end{array}\right.$ & $\begin{array}{r}7 \\
2 \\
17 \\
10 \\
8\end{array}$ & $\begin{aligned} 6 / 7 & =86 \% \\
2 / 2 & =100 \% \\
16 / 20 & =80 \% \\
10 / 12 & =83 \% \\
6 / 8 & =75 \%\end{aligned}$ \\
\hline Sex & $\left\{\begin{array}{l}\mathbf{M} \\
\mathbf{F}\end{array}\right.$ & $\begin{array}{l}34 \\
10\end{array}$ & $\begin{aligned} 32 / 37 & =86 \% \\
8 / 12 & =67 \%\end{aligned}$ \\
\hline $\begin{array}{l}\text { Heart weight } \\
\text { (g) }\end{array}$ & $\left\{\begin{array}{l}>500 \\
\leqslant 500\end{array}\right.$ & $\begin{array}{l}17 \\
27\end{array}$ & $\begin{array}{l}16 / 19=84 \% \\
24 / 30=80 \%\end{array}$ \\
\hline \multicolumn{2}{|c|}{$\begin{array}{l}\text { Diabetes mellitus } \\
\text { Treated }{ }^{\star} \\
\text { Untreated }\end{array}$} & $\begin{array}{r}7 \\
37\end{array}$ & $\begin{aligned} 5 / 8 & =63 \% \\
35 / 41 & =85 \%\end{aligned}$ \\
\hline \multicolumn{2}{|l|}{ All patients } & 44 & $40 / 49=82 \%$ \\
\hline
\end{tabular}

Two recent thrombi localised in old recanalised occlusions are excluded.

$\star$ Patients who, according to the case records, were treated with diet, tablets, or insulin.

occurred with pre-existing stenoses of less than $75 \%$ ( 1 of 39 cases $(3 \%)$ ).

Excluding two cases of rethrombosed old recanalised occlusions, an underlying ruptured atheromatous plaque was identified in 40 of $49(82 \%)$ recently thrombosed coronary artery segments, and there was no correlation with age, sex, heart weight, or diabetes mellitus (Table 2).

The ruptured fibrous cap was always very thin and usually heavily infiltrated with foam cells. In 16 of the 40 thrombi $(40 \%)$ precipitated by plaque rupture, fragments of the disrupted plaque were found intimately mixed with and often deeply buried in the centre of the thrombus (Fig. 3 and 4).

At the site of rupture aggregated platelets could be identified readily by their characteristic morphology in all thrombi less than a few days old; later on the platelets were condensed into a homogeneous eosinophilic mass. In the 11 cases where an underlying plaque rupture was not identified, minor intimal irregularities or breaks were usually seen. The pre- existing stenosis at the site of thrombosis was greater than $95 \%$ in the majority of these cases (Table 3).

\section{Discussion}

Pathologists have been aware for many years that the thin fibrous "cap" separating the fatty material of an atheromatous plaque from the lument of an artery may rupture. ${ }^{125}$ When this happens, the following events may occur separately, simultaneously, or successively: (1) haemorrhage into the plaque ${ }^{22} 23$; (2) discharge into the lumen of atheromatous material causing local vascular obstruction or peripheral embolisation ${ }^{13} 25$; (3) mural thrombosis ${ }^{14}$; (4) occlusive thrombosis. 6-12 $^{-12}$

Intimal haemorrhage alone usually causes no appreciable acute deterioration of the luminal narrowing and is, therefore, probably clinically silent. No instance of so-called occluding intimal haematoma ${ }^{\mathrm{s}}$ was seen in this study. Mural thrombosis and peripheral embolisation may cause symptoms such as unstable angina while occlusive thrombosis is always a life-threatening event and is the main cause of acute myocardial infarction. 2627

The exact connection between plaque rupture, chronic atherosclerotic stenoses, intimal haemorrhage, and luminal thrombosis has not previously been reported. This investigation shows that the fate of a plaque rupture depends on the degree of preexisting luminal narrowing. If the stenosis is less than $75 \%$ the rupture will cause intimal haemorrhage without significant luminal thrombosis, but with stenoses in excess of $75 \%$ occlusive thrombus formation occurs with increasing frequency. Thus, rupture of a plaque causing 90 to $95 \%$ stenosis usually results in occlusive thrombus formation, and rupture of one which had caused more than $95 \%$ stenosis seems always to do so. As angiographic assessment of luminal narrowing compared with histological determination always underrates the severity of stenosis, though to a variable degree (because of different areas of reference used by clinicians and pathologists 24 ), it can be deduced that angiographically assessed lesions with greater than $90 \%$ luminal area reduction nearly always thrombose if the stenotic plaque ruptures.

Table 3 Degree of pre-existing atherosclerotic stenosis at site of occlusive thrombosis and presence or absence of underlying plaque rupture

\begin{tabular}{|c|c|c|c|c|c|c|c|}
\hline & \multicolumn{7}{|c|}{ Histological stenosis (\%) } \\
\hline & $\overline{70-74}$ & $75-79$ & $80-84$ & $85-89$ & $90-94$ & $95-99$ & Total \\
\hline $\begin{array}{l}\text { Plaque rupture } \\
\text { Absent } \\
\text { Present } \\
\text { Total }\end{array}$ & $\begin{array}{l}1 \\
1\end{array}$ & $\begin{array}{l}1 \\
2 \\
3\end{array}$ & $\begin{array}{l}2 \\
2\end{array}$ & $\begin{array}{l}1 \\
8 \\
9\end{array}$ & $\begin{array}{r}3 \\
15 \\
18\end{array}$ & $\begin{array}{r}6 \\
12 \\
18\end{array}$ & $\begin{array}{l}11 \\
40 \\
51\end{array}$ \\
\hline
\end{tabular}

Four fatal mural thrombi of the left main stem are included: two without (pre-existing stenosis of 78 and $97 \%$, respectively) and two with an underlying plaque rupture (pre-existing stenosis of 78 and $86 \%$, respectively). 
Step-sectioning of coronary thrombi always or nearly always shows an underlying acute vascular lesion, most often a ruptured atheromatous plaque. ${ }^{6-12}$ The frequent finding of plaque contents widely dispersed deeply in the thrombus ${ }^{725}$ clearly indicates that plaque rupture has preceded and not resulted from thrombus formation. A second point of evidence of the primary role of plaque rupture in the thrombogenesis is the nature of the thrombus at the site of rupture; it consists almost entirely of aggregated platelets. ${ }^{6812}$

The platelet response in the vascular lumen is thus secondary to local stimuli from the vascular wall, and the degree of stenosis (local flow disturbances) is decisive for whether the platelet nidus at the site of rupture remains mural and limited or evolves into an occlusive thrombus. No doubt platelets are crucial for arterial thrombus formation, but in the majority of cases there is no need to suggest intrinsic abnormalities of platelet function ${ }^{16}{ }^{17}$ or some precipitating thrombotic/hypercoagulable state ${ }^{1819}$ to explain the sequence of events leading to the final thrombotic occlusion of an atherosclerotic coronary artery. In the few cases, however, where no precipitating plaque rupture is identified it is possible that a thrombotic propensity in combination with severe stenosis is more important.

It has been claimed that plaque rupture is the underlying fatal coronary event in older rather than younger persons, ${ }^{25}$ and it has been proposed that plaque rupture could constitute a link between hypertension and coronary thrombosis. ${ }^{3}$ Neither of these connections could be confirmed in the present study where no special correlation was found between fatal plaque rupture and age, cardiac hypertrophy (hypertension), sex, or diabetes mellitus. Rather, plaque rupture with severe pre-existing stenosis seems to constitute a kind of final common pathway in the atherosclerotic process causing the ultimate occlusion of the lumen by a thrombus.

The mechanism underlying the sudden rupture of a soft atheromatous plaque has been disputed. Probably plaque rupture is an incidental event in the evolution and growth of the atherosclerotic plaque and happens when the process of softening has gone so far that just a thin cap of fibrous tissue separates the fatty "gruel" from the lumen. This attenuated fibrous cap is often heavily infiltrated with foam cells, ${ }^{811}$ and probably no more than the normal bending and twisting of the arteries which occur during every heart contraction are needed to tear such a thin and fragile cap. Maybe even normal haemodymanic stresses induced by the pulsatile flow and by variations in the blood pressure $^{610}$ or by changes in the vascular tone (spasm?) 2528 are sufficient. Thus, plaque rupture is probably a random event which in principle can be prevented only by arresting the atherosclerotic process in the vascular wall. Nevertheless, recent experimental ${ }^{29}$ and clinical ${ }^{30}$ observations make it likely that the complicating (and life-threatening) thrombotic response in the vascular lumen can be suppressed to some degree by appropriate inhibition of platelet function.

I thank Dr Preben Johansen, Aalborg Hospital, and Dr Jens Vilhelm Thorborg and Jens Sand Kristensen, Randers Hospital, for critical review of the manuscript.

\section{References}

1 Clark E, Graef I, Chasis H. Thrombosis of the aorta and coronary arteries. Archives of Pathology 1936; 22: 183212.

2 Woolf N. Thrombosis and atherosclerosis. In: Bloom AL, Thomas DP, eds. Haemostasis and thrombosis. Edinburgh, London, Melbourne, New York: Churchill Livingstone, 1981: 527-53.

3 Crawford T. Pathology of ischaemic heart disease. London, Boston: Butterworths, 1977: 47-52.

4 Paterson JC. Capillary rupture with intimal hemorrhage as a causative factor in coronary thrombosis. Archives of Pathology 1938; 25: 474-87.

5 Wartman WB. Occlusion of the coronary arteries by hemorrhage into their walls. Am Heart $\mathcal{F}$ 1938; 15: 459 70.

6 Constantinides P. Plaque fissures in human coronary thrombosis. Fournal of Atherosclerosis Research 1966; 6: 1-17.

7 Chapman I. Morphogenesis of occluding coronary artery thrombosis. Arch Pathol 1965; 80: 256-61.

8 Friedman M, Van den Bovenkamp GJ. The pathogenesis of a coronary thrombus. Am F Pathol 1966; 48: 19-44.

9 Sinapius D. Beziehungen zwischen Koronarthrombosen und Myokardinfarkten. Dtsch Med Wochenschr 1972; 97: 443-8.

10 Ridolfi RL, Hutchins GM. The relationship between coronary artery lesions and myocardial infarcts: ulceration of atherosclerotic plaques precipitating coronary thrombosis. Am Heart f 1977: 93: 468-86.

11 Horie T, Sekiguchi M, Hirosawa K. Coronary thrombosis in pathogenesis of acute myocardial infarction. Histopathological study of coronary arteries in 108 necropsied cases using serial section. Br Heart F 1978; 40: 15361.

12 Davies MJ, Thomas T. The pathological basis and microanatomy of occlusive thrombus formation in human coronary arteries. Philos Trans $R$ Soc Lond [Biol] 1981; 294: 225-9.

13 Crawford T, Dexter D, Teare RD. Coronary-artery pathology in sudden death from myocardial ischaemia. A comparison by age-groups. Lancet 1961; i: 181-5.

14 Sinapius D. Uber Wandveränderungen bei Coronarthrombose. Bemerkungen zur Häufigkeit, Entstehung und Bedeutung. Klin Wochenschr 1965; 43: 875-80.

15 Rentrop P, Blanke H, Karsch KR, Kaiser H, Köstering $H$, Leitz $K$. Selective intracoronary thrombolysis in 
acute myocardial infarction and unstable angina pectoris. Circulation 1981; 63: 307-17.

16 Hampton JR, Gorlin R. Platelet studies in patients with coronary artery disease and in their relatives. $\mathrm{Br} \mathrm{Heart} \mathcal{F}$ 1972; 34: 465-71.

17 Wu KK, Hoak JC. Spontaneous platelet aggregation in arterial insufficiency: mechanisms and implications. Thromb Haemostas 1976; 35: 702-11.

18 Meade TW, Chakrabarti R, Haines AP, et al. Haemostatic function and cardiovascular death: early results of a prospective study. Lancet 1980; i: $1050-4$.

19 Kostis JB, Baughman DJ, Kuo PT. Association of recurrent myocardial infarction with hemostatic factors. A prospective study. Chest 1982; 81: 571-5.

20 Lowe GDO, Stromberg P, Forbes CD, McArdle BM, Lorimer AR, Prentice CRM. Increased blood viscosity and fibrinolytic inhibitor in type II hyperlipoproteinaemia. Lancet 1982; i: 472-5.

21 Arkin CF, Hartman AS. The hypercoagulability states. CRC Crit Rev Clin Lab Sci 1979; 10: 397-429.

22 Drury RAB. The role of intimal haemorrhage in coronary occlusion. Fournal of Pathology and Bacteriology 1954; 67: 207-15.

23 Friedman M, Van den Bovenkamp G. The pathogenesis of coronary intramural haemorrhages. Br f Exp Pathol 1966; 47: 347-55.

24 Falk E. Coronary artery narrowing without irreversible myocardial damage or development of collaterals.
Assessment of "critical" stenosis in a human model. $\mathrm{Br}$ Heart f 1982; 48: 265-71.

25 Leary T. Coronary spasm as a possible factor in produc: ing sudden death. Am Heart $\mathcal{f}$ 1934; 10: 338-44.

26 DeWood MA, Spores J, Notske R, et al. Prevalence of total coronary occlusion during the early hours of transmural myocardial infarction. $N$ Engl f Med 1980; 303: 897-902.

27 Davies MJ, Fulton WFM, Robertson WB. The relation of coronary thrombosis to ischaemic myocardial necrosis. f Pathol 1979; 127: 99-110.

28 Hellstrom HR. Evidence in favor of the vasospastic cause of coronary artery thrombosis. Am Heart $\mathcal{F} 1979$; 97: $449-52$.

29 Folts JD, Gallagher K, Rowe GG. Blood flow reductions in stenosed canine coronary arteries: vasospasm or platelet aggregation? Circulation 1982; 65: 248-55.

30 Chesebro JH, Clements IP, Fuster V, et al. A plateletinhibitor-drug trial in coronary-artery bypass operations: benefit of perioperative dipyridamole and aspirin therapy on early post-operative vein-graft patency. $N$ Engl $f$ Med 1982; 307: 73-8.

Requests for reprints to Dr Erling Falk, Institute of Pathology, Aalborg Sygehus, Nord, DK-9000 Aalborg, Denmark. 